This information is current as of April 26, 2023.

\title{
Regional Cerebral Blood Flow Using Quantitative MR Angiography
}

M. Zhao, S. Amin-Hanjani, S. Ruland, A.P. Curcio, L. Ostergren and F.T. Charbel

AJNR Am J Neuroradiol 2007, 28 (8) 1470-1473

doi: https://doi.org/10.3174/ajnr.A0582

http://www.ajnr.org/content/28/8/1470 


\section{ORIGINAL RESEARCH \\ Regional Cerebral Blood Flow Using Quantitative MR Angiography}

\author{
M. Zhao \\ S. Amin-Hanjani \\ S. Ruland \\ A.P. Curcio \\ L. Ostergren \\ F.T. Charbel
}

BACKGROUND AND PURPOSE: We sought to derive regional cerebral blood flow using vessel flows from quantitative MR angiography (qMRA).

\begin{abstract}
MATERIALS AND METHODS: Flow rates in the 15 major cerebral arteries were measured on retrospectively gated fast 2D phase-contrast MR angiography obtained in 83 healthy adult volunteers (age range, 24-74 years; mean, 42 years). The arterial network of the brain was partitioned into 12 different regions, in which flows were calculated from the measured flows of the 15 cerebral arteries.
\end{abstract}

\begin{abstract}
RESULTS: The mean flows of the 15 arteries and the 12 regions were calculated. The mean total cranial flow and the mean total cerebral blood flow were $949 \pm 158 \mathrm{~mL} / \mathrm{min}$ and $695 \pm 113 \mathrm{~mL} / \mathrm{min}$, respectively. The mean regional flows for the anterior and posterior circulation were $483 \pm 87 \mathrm{~mL} / \mathrm{min}$ and $212 \pm 34 \mathrm{~mL} / \mathrm{min}$, respectively. The relative contributions of the flows in the 11 regions to their parent regions were obtained. The mean flows in the individual arteries and the regions with age were also calculated. The mean flows for the female group were significantly lower than those for the male group $(P<.001)$ for the 2 common carotids and the cranial circulation and left/right extracranial circulation. However, the intracranial circulation was not different between sexes.
\end{abstract}

CONCLUSIONS: The 12 regions in the cerebral circulation were identified and formed into a partition tree, and the mean regional flow for each region was determined using vessel flows from qMRA.
Q uantification of blood flow to the brain may be useful for distinguishing patients at risk for cerebral ischemia caused by hemodynamic compromise. Hemodynamic assessment by quantitative MR angiography (qMRA) has been used to identify patients at high risk for stroke and to guide treatment decisions. ${ }^{1,2}$ qMRA has been used to determine the total cerebral blood flow (CBF), ${ }^{3-5}$ the effect of age and sex on the total $\mathrm{CBF},{ }^{6}$ distribution of $\mathrm{CBF}$ in the circle of Willis, ${ }^{7}$ and cerebral autoregulation, ${ }^{8}$ as well as to evaluate various cerebrovascular disorders. ${ }^{9-16}$ The range of blood flow for a healthy individual vessel in the brain, however, can be quite diverse because of inherent vascular anatomy and/or anatomic variations in the circle of Willis. ${ }^{7}$ A decreased flow in an individual vessel may not necessarily be caused by vascular disease. An effective decision-making paradigm based on hemodynamic assessment requires an algorithm that accounts for both anatomic variations and assessment of flows in distal vessels (regional CBF). ${ }^{1}$ However, current methods for assessing regional CBF can be technically difficult, time consuming, and may subject the patient to ionizing radiation. ${ }^{10,11,17}$ The purpose of this present study was to determine the flows to specific brain areas, that is, regional cerebral flow using individual vessel flows obtained from qMRA.

\section{Methods}

\section{$q M R A$}

qMRA was performed on either a 1.5T or 3T MR imager (Excite; GE Healthcare, Milwaukee, Wis). The MR volume flow measurements

Received July 24, 2006; accepted after revision January 6, 2007.

From the Departments of Neurosurgery (M.Z., S.A.-H., F.T.C.) and Neurology (S.R.), University of Illinois at Chicago, Chicago, III; and VasSol (M.Z., A.P.C., L.O.), Chicago, III.

This work was generously supported by the Falk Foundation.

Please address correspondence to Meide Zhao, Neuropsychiatric Institute (MC 799), Department of Neurosurgery, University of Illinois at Chicago, 912 South Wood St, Chicago, IL 60612-5970; e-mail: mzhao@uic.edu

DOI 10.3174/ajnr.A0582 were calculated from NOVA software (VasSol, Chicago, Ill) on a separate $\mathrm{PC}$ workstation. The protocol that uses a retrospectively gated fast $2 \mathrm{D}$ phase-contrast sequence has been described previously. ${ }^{1,18,19}$

The protocol entails performing a standard axial 2D and 3D timeof-flight (TOF) MR angiography (MRA) of the cervical and cranial vasculature, similar to conventional neck and head MRA. The acquired TOF images were then transmitted to a workstation where rotating 3D surface-rendered vascular images (Figs 1 and 2) were reconstructed using a marching-cube algorithm. ${ }^{18}$ Optimal perpendicular scan plane determination was based on the scan line calculated by a line-fitting algorithm introduced by Zhao et al. ${ }^{18}$ The coordinates obtained specify the position of an oblique fast $2 \mathrm{D}$ phasecontrast sequence, which was then performed based on these coordinates, using a retrospectively gated $2 \mathrm{D}$ phase-contrast sequence with the following imaging parameters: TR, 10-15 ms; TE, 4-7 ms; flip angle, 15; number of excitations, 4; section thickness, 5 $\mathrm{mm}$ for neck vessels and $3 \mathrm{~mm}$ for intracranial vessels; FOV, $180 \mathrm{~mm}$ for neck vessels and $140 \mathrm{~mm}$ for intracranial vessels; and matrix, $256 \times 128$ for neck vessels and $256 \times 192$ for intracranial vessels. Velocity encoding was automatically adjusted with the NOVA software if necessary. The acquired phase-contrast images were transferred to the workstation for flow quantification. A region of interest was automatically placed on the phase-contrast images and was also displayed in the 3D surface-rendered image for vessel verification. The vessel borders over a cardiac cycle were automatically extracted and displayed on the color-coded and magnified region of interest image for vessel border verification. The velocities at all of the pixels inside the vessel border were then integrated to calculate the flow in milliliters per minute. The flows were averaged over a cardiac cycle to obtain the mean flow for each vessel. The total scan time necessary for the entire qMRA study was $30-45$ minutes. This protocol has been used routinely for the evaluation of patients with cerebrovascular diseases. $^{1,20}$

The flow measurement accuracy using the protocol has been extensively tested with phantom blood flow simulators. ${ }^{19}$ For steady flow conditions, the average variation from actual measurements was only $4.1 \% \pm 3.0 \%$. For pulsatile flow conditions, the variation was 


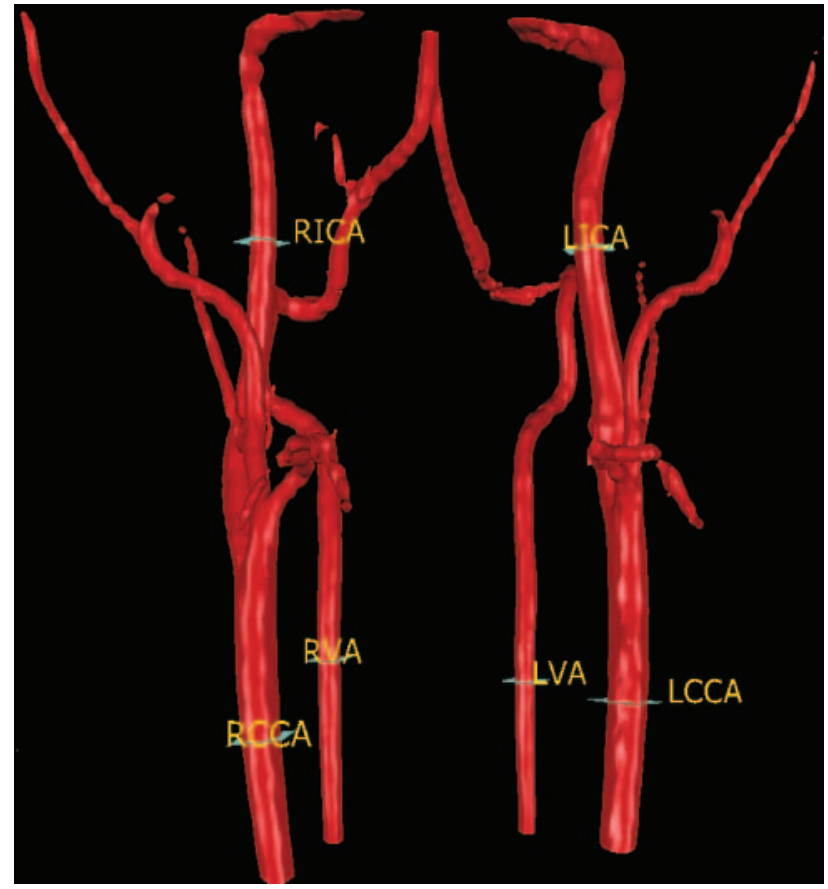

Fig 1. The 6 section planes showing where the flow measurements were made for the 6 neck vessels.

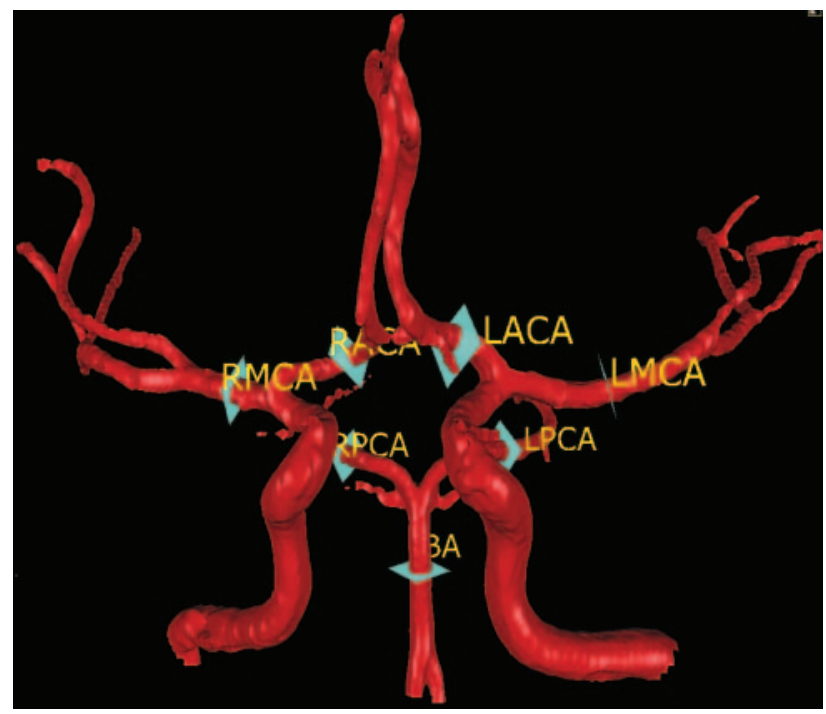

Fig 2. The 7 section planes showing where the flow measurements were made for the 7 head vessels.

only $4.5 \% \pm 3.1 \% .{ }^{19}$ The intrarater reliability has been investigated by repeated measurements in adult subjects. Coefficients of variation were within $5 \%$ for 9 vessels and $5 \%-10 \%$ for 3 vessels. ${ }^{21}$ Vessel measurement variation was also examined by performing 2 consecutive full 13-vessel scans, removing the subject from the scanner between scans. ${ }^{22}$ Mean coefficients of variation were less than $5 \%$ for 7 vessels and $5 \%-10 \%$ for 5 vessels. ${ }^{22}$ The interrater reliability was investigated by measuring flows from different users on 2 different vessels (11 different operators were used for the basilar artery; 9 different operators were used for the left middle cerebral artery). Mean coefficients of variation were less than $4 \%$ for both vessels (data on file at VasSol).

The measured vessels include 6 vessels in the neck, 7 vessels in the head, and the left and right posterior communicating artery (PcomA;

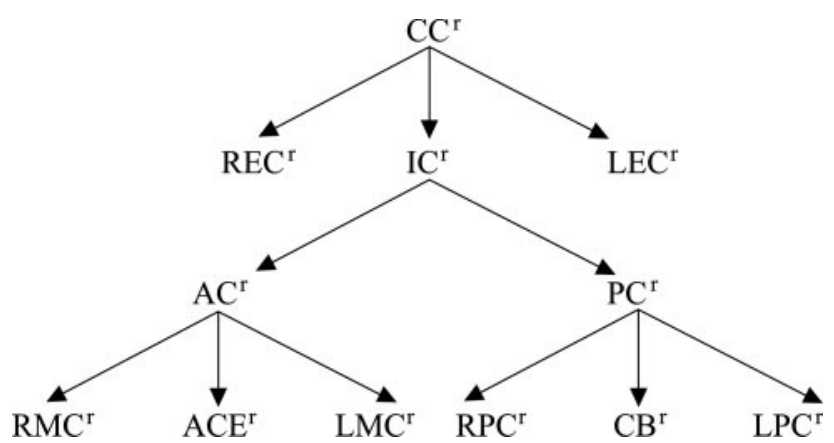

Fig 3. Partition tree of the cerebral circulation.

LPcomA and RPcomA) if present. The 6 vessels in the neck include the left and the right common carotid arteries (LCCA and RCCA), the left and the right internal carotid arteries (LICA and RICA), and the left and the right vertebral arteries (LVA and RVA). Figure 1 depicts the locations of the neck vessel measurements. The 7 vessels in the head include the basilar artery, the M1 segment of the left and the right middle cerebral arteries, the A1 segment of the left and the right anterior cerebral arteries, and the P2 segment of the left and the right posterior cerebral arteries. Figure 2 depicts the locations of the intracranial vessel measurements. The measurements for both the flow rate and the flow direction for each vessel, as well as the vascular anatomy of both head and neck, were assessed on a PC workstation by a physician.

\section{Partition Algorithm}

Cranial circulation $\left(\mathrm{CC}^{\mathrm{r}}\right)$ is the circulation in cranial region (where " $\mathrm{r}$ " in $\mathrm{CC}^{\mathrm{r}}$ stands for region). $\mathrm{CC}^{\mathrm{r}}$ consists of the right extracranial circulation $\left(\mathrm{REC}^{\mathrm{r}}\right)$, the left extracranial circulation $\left(\mathrm{LEC}^{\mathrm{r}}\right)$, and the intracranial circulation $\left(\mathrm{IC}^{\mathrm{r}}\right)$. $\mathrm{IC}^{\mathrm{r}}$ consists of the anterior circulation $\left(\mathrm{AC}^{\mathrm{r}}\right)$ and the posterior circulation $\left(\mathrm{PC}^{\mathrm{r}}\right) . \mathrm{AC}^{\mathrm{r}}$ consists of the right middle cerebral region $\left(\mathrm{RMC}^{\mathrm{r}}\right)$, the left middle cerebral region $\left(\mathrm{LMC}^{\mathrm{r}}\right)$, and the anterior cerebral region $\left(\mathrm{ACA}^{\mathrm{r}}\right) \cdot \mathrm{PC}^{\mathrm{r}}$ consists of the right posterior cerebral region $\left(\mathrm{RPC}^{\mathrm{r}}\right)$, the left posterior cerebral region $\left(\mathrm{LPC}^{\mathrm{r}}\right)$, and the cerebellar-basilar region $\left(\mathrm{CB}^{\mathrm{r}}\right)$. The partitions of the $\mathrm{CC}^{\mathrm{r}}$ were formed into a partition tree (Fig 3). A parent region can have 1 or more than 1 subregion. $\mathrm{CC}^{\mathrm{r}}$ is the parent region for $\mathrm{LEC}^{\mathrm{r}}$, $R E C^{r}$, and $I^{r}$. IC ${ }^{r}$ is the parent region for $A C^{r}$ and $P C^{r} . A C^{r}$ is the parent region for $\mathrm{LMC}^{\mathrm{r}}, \mathrm{RMC}^{\mathrm{r}}$, and $\mathrm{ACA}^{\mathrm{r}}$. $\mathrm{PC}^{\mathrm{r}}$ is the parent region for $\mathrm{LPC}^{\mathrm{r}}, \mathrm{RPC}^{\mathrm{r}}$, and $\mathrm{CB}^{\mathrm{r}}$.

The flow to each of the 12 regions was calculated as follows. $\mathrm{CC}^{\mathrm{r}}$ flow was determined by summing the flows of the 4 inlet vessels to the brain, that is, LCCA, RCCA, LVA, and RVA. The IC ${ }^{\mathrm{r}}$ flow was determined by summing the flows of the 4 inlet vessels to IC ${ }^{\mathrm{r}}$, that is, LICA, RICA, LVA, and RVA, which is the mean total CBF. LEC ${ }^{\mathrm{r}}$ (or REC ${ }^{\mathrm{r}}$ ) flow was determined by subtracting the LICA (or RICA) flow from the LCCA (or RCCA) flow. AC ${ }^{\mathrm{r}}$ flow was obtained as follows: summing the LICA and RICA flows, subtracting any fetal PCA or posterior directed PcomA flows. Alternatively, anterior directed PcomA flow would be added. $\mathrm{PC}^{\mathrm{r}}$ flow was obtained by subtracting $\mathrm{AC}^{\mathrm{r}}$ flow from $\mathrm{IC}^{\mathrm{r}}$ flow. The $\mathrm{ACA}^{\mathrm{r}}$ flow was calculated by summing the left and right anterior cerebral region flows (valid only for healthy volunteers, otherwise flow direction should be considered). The $\mathrm{CB}^{\mathrm{r}}$ flow was determined by subtracting the sum of $\mathrm{LPC}^{\mathrm{r}}$ and $\mathrm{RPC}^{\mathrm{r}}$ flow from the $\mathrm{PC}^{\mathrm{r}}$ flow. 
Table 1: Flow rates in the fifteen arteries

\begin{tabular}{lllllllllllllll}
\hline LCCA & LVA & LICA & LMCA & LACA & LPCA & LPcomA & BA & RCCA & RVA & RICA & RMCA & RACA & RPCA & RPcomA
\end{tabular}

$\begin{array}{llllllllllllllll}389 \pm 73 & 96 \pm 38 & 264 \pm 52 & 150 \pm 31 & 85 \pm 26 & 66 \pm 14 & 17 \pm 25 & 131 \pm 40 & 381 \pm 79 & 83 \pm 32 & 252 \pm 52 & 145 \pm 27 & 80 \pm 28 & 63 \pm 14 & 15 \pm 22\end{array}$

Note:-Data are mean \pm SD in milliliters per minute.

Table 2: Regional cerebral blood flows in the cerebral circulation

\begin{tabular}{lcccccccccccc}
\hline CC $^{r}$ & LEC $^{r}$ & AC $^{r}$ & LMC $^{r}$ & ACE $^{r}$ & LPC $^{r}$ & IC $^{r}$ & REC $^{r}$ & PC $^{r}$ & RMC $^{r}$ & CB $^{r}$ & RPC $^{r}$ \\
\hline $949 \pm 158$ & $126 \pm 61$ & $483 \pm 87$ & $150 \pm 31$ & $187 \pm 50$ & $66 \pm 14$ & $695 \pm 113$ & $129 \pm 63$ & $212 \pm 34$ & $145 \pm 27$ & $82 \pm 26$ & $63 \pm 14$
\end{tabular}

Note:-Data are mean \pm SD in milliliters per minute.

\section{Subjects and Data Analysis}

Ninety-two healthy adult volunteers (age range, 21-74 years; mean age, 42 years; 43 men and 49 women) without history of cerebrovascular disease underwent qMRA of head and neck vessels. Subjects gave informed written consent before participating. The institutional review board of the University of Illinois at Chicago approved the study protocol.

Flows were expressed as mean $\pm \mathrm{SD}$. The differences in age between men and women were tested using a Student independent $t$ test. Paired Student $t$ test was used to determine the differences between flows for the left and right arteries (or regions). The effect of age on flows for individual vessels and regions was evaluated using linear regression analysis. In all of the tests, $P<.05$ was considered statistically significant. All of the analyses were performed with Analyze-it (1.71, Analyze-it Software, England).

\section{Results}

Of 92 subjects, 9 were excluded because of subject motion $(n=1)$, missed RACA measurement $(n=1)$, and missed PcomA measurements (LPcomA, RPcomA, or both PcomAs; $n=7$ ). Eighty-three subjects (age range, 24-74 years; mean age, 42 years; 40 men and 43 women) had adequate QMRA studies and were included in the data analysis. Among the 83 subjects, 7 subjects had unilateral fetal PCAs (8\%), 3 had bilateral fetal PCAs (4\%), 1 had persistent trigeminal artery (1\%), 1 had absent RACA (1\%), 28 had no PcomAs (34\%), 22 had both PcomAs (26\%), and 33 had only 1 PcomA (40\%). The flow directions in the PcomAs were posterior in all but 1 subject. Nine subjects had very short M1 segments (11\%) where the flows of the $2 \mathrm{M} 2$ branches were measured and summed to derive the flow of the M1 segment.

The mean flows of the 15 arteries and the 12 regions are shown in Tables 1 and 2, respectively. The mean flows were as follows: $\mathrm{CC}^{\mathrm{r}}, 949 \pm 158 \mathrm{~mL} / \mathrm{min}$; $\mathrm{IC}^{\mathrm{r}}, 695 \pm 113 \mathrm{~mL} / \mathrm{min}$; $\mathrm{AC}^{\mathrm{r}}, 483 \pm 87 \mathrm{~mL} / \mathrm{min}$; and $\mathrm{PC}^{\mathrm{r}}, 212 \pm 34 \mathrm{~mL} / \mathrm{min}$. The relative flow contribution in each of the 11 regions to their parent regions is shown in Fig 4. The relative contribution from the 3 subregions to $\mathrm{CC}^{\mathrm{r}}$ was $74 \% \pm 9 \%$ for $\mathrm{IC}^{\mathrm{r}}, 13 \% \pm$ $5 \%$ for $\mathrm{LEC}^{\mathrm{r}}$, and $13 \% \pm 5 \%$ for REC $\mathrm{r}$. The relative contribution to $\mathrm{IC}^{\mathrm{r}}$ from its 2 subregions was $69 \% \pm 3 \%$ for $\mathrm{AC}^{\mathrm{r}}$ and $31 \% \pm 3 \%$ for $\mathrm{PC}^{\mathrm{r}}$. The relative contribution to $\mathrm{AC}^{\mathrm{r}}$ from its 3 subregions was $31 \% \pm 4 \%$ for $\mathrm{LMC}^{\mathrm{r}}, 30 \% \pm 3 \%$ for $\mathrm{RMC}^{\mathrm{r}}$, and $39 \% \pm 6 \%$ for $\mathrm{ACA}^{\mathrm{r}}$. The relative contribution to $\mathrm{PC}^{\mathrm{r}}$ from its 3 subregions was $32 \% \pm 5 \%$ for $\mathrm{LPC}^{\mathrm{r}}, 30 \% \pm 5 \%$ for $\mathrm{RPC}^{\mathrm{r}}$, and $38 \% \pm 9 \%$ for $\mathrm{CB}^{\mathrm{r}}$.

Table 3 shows the effect of age on the mean flows for individual arteries and regions. Overall, both total cranial flow $\left(\mathrm{CC}^{\mathrm{r}}\right)$ and total cerebral flow $\left(\mathrm{IC}^{\mathrm{r}}\right)$ declined with age $(P<.02)$, from $1047 \pm 178 \mathrm{~mL} / \mathrm{min}\left(\mathrm{CC}^{\mathrm{r}}\right)$ and $788 \pm 102 \mathrm{~mL} / \mathrm{min}\left(\mathrm{IC}^{\mathrm{r}}\right)$

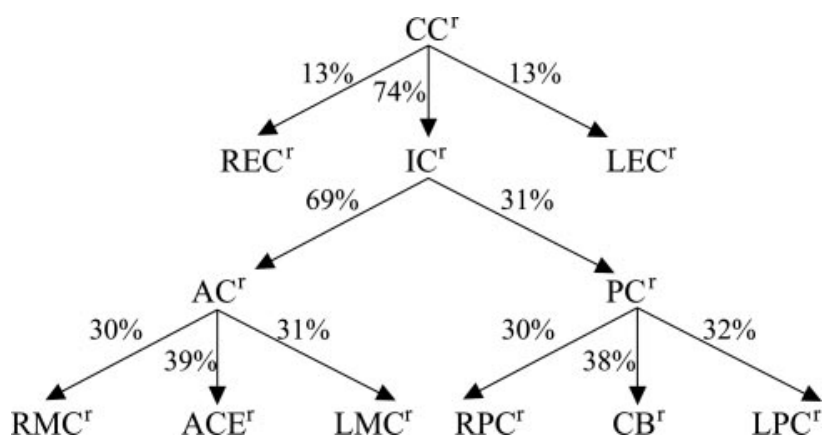

Fig 4. The relative contribution of flows in each of 11 regions to its parent region.

\begin{tabular}{|c|c|c|c|}
\hline $\begin{array}{l}\text { Artery or } \\
\text { Region }\end{array}$ & $P$ & $\begin{array}{c}\text { Youngest } \\
\text { Group, } \\
\text { mL/min }\end{array}$ & $\begin{array}{l}\text { Oldest } \\
\text { Group, } \\
\text { mL/min }\end{array}$ \\
\hline LCCA & $<.01$ & $423 \pm 86$ & $349 \pm 51$ \\
\hline LVA & $<.01$ & $125 \pm 35$ & $69 \pm 39$ \\
\hline LICA & $<.03$ & $296 \pm 45$ & $221 \pm 49$ \\
\hline BA & $<.03$ & $164 \pm 25$ & $118 \pm 15$ \\
\hline LACA & $<.01$ & $101 \pm 28$ & $67 \pm 20$ \\
\hline $\mathrm{CC}^{\mathrm{r}}$ & $<.02$ & $1047 \pm 178$ & $905 \pm 129$ \\
\hline$I^{r}$ & $<.02$ & $788 \pm 102$ & $621 \pm 71$ \\
\hline$A C^{r}$ & $<.02$ & $559 \pm 74$ & $433 \pm 52$ \\
\hline$P C^{r}$ & $<.03$ & $229 \pm 32$ & $188 \pm 23$ \\
\hline
\end{tabular}

Note:- LCCA indicates left common carotid artery; LVA, left vertebral artery; LICA, lef internal carotid artery; BA, basilar artery; LACA, left anterior cerebral region; $C^{r}$, cranial circulation region; $\mathrm{IC}^{\mathrm{r}}$, intracranial circulation region; $\mathrm{AC}^{\mathrm{r}}$, anterior circulation region; $\mathrm{PC}^{\mathrm{r}}$ posterior circulation region.

\begin{tabular}{|c|c|c|c|c|}
\hline $\begin{array}{l}\text { Artery or } \\
\text { Region }\end{array}$ & $\begin{array}{c}\text { Flow } \\
\text { (Female), } \\
\mathrm{mL} / \mathrm{min}\end{array}$ & $\begin{array}{c}\text { Flow } \\
\text { (Male), } \\
\mathrm{mL} / \mathrm{min}\end{array}$ & Hypotheses & $P$ \\
\hline LCCA & $366 \pm 64$ & $414 \pm 73$ & $\mathrm{~F} \leq \mathrm{M}$ & $<.001$ \\
\hline RCCA & $345 \pm 56$ & $419 \pm 82$ & $\mathrm{~F} \leq \mathrm{M}$ & $<.0001$ \\
\hline $\mathrm{CC}^{\mathrm{r}}$ & $897 \pm 139$ & $1006 \pm 161$ & $\mathrm{~F} \leq \mathrm{M}$ & $<.001$ \\
\hline LEC $^{r}$ & $99 \pm 53$ & $154 \pm 56$ & $\mathrm{~F} \leq \mathrm{M}$ & $<.0001$ \\
\hline REC & $96 \pm 41$ & $164 \pm 65$ & $\mathrm{~F} \leq \mathrm{M}$ & $<.0001$ \\
\hline
\end{tabular}

Note:- LCCA indicates left common carotid artery; RCCA, right common carotid artery $\mathrm{CC}^{r}$, cranial circulation region; $\mathrm{LEC}^{r}$, left extracranial circulation; $\mathrm{REC}^{r}$, right extracranial circulation. $\mathrm{F} \leq \mathrm{M}$ (or $\mathrm{F} \geq \mathrm{M}$ ) indicates that the flow for the female group is lower (or higher) than the flow for male group.

for the youngest group (age $24-30$ years; $n=12$ ) to $905 \pm 129$ $\mathrm{mL} / \mathrm{min}\left(\mathrm{CC}^{\mathrm{r}}\right)$ and $621 \pm 71 \mathrm{~mL} / \mathrm{min}\left(\mathrm{IC}^{\mathrm{r}}\right)$ for the oldest group (age $61-74$ years; $n=4$ ).

Table 4 shows the sex difference of the mean volume flows for individual arteries and regions. The mean flows for the female group were lower than the male group $(P<.001)$ for 
the total CC $^{\mathrm{r}}$, the 2 common carotids (LCCA and RCCA), and the LEC $^{\mathrm{r}}$ and REC ${ }^{\mathrm{r}}$. However, the IC ${ }^{\mathrm{r}}$ was not different between sexes.

\section{Discussion}

In this study, we partitioned the cerebral arterial network into 12 different regions. We calculated the regional CBF using the measured flows of the 15 cerebral arteries as obtained by qMRA. Based on these measurements in subjects without history of cerebrovascular disease, the SDs of the relative contribution of the $\mathrm{AC}^{\mathrm{r}}$ and $\mathrm{PC}^{\mathrm{r}}$ to the $\mathrm{IC}^{\mathrm{r}}$ are small $(3 \%)$. The SDs of the relative contribution of the $\mathrm{LMC}^{\mathrm{r}}$ and $\mathrm{RMC}^{\mathrm{r}}$ to the $\mathrm{AC}^{\mathrm{r}}$ are also small (4\% and 3\%, respectively). Unlike individual vessel flows that are subject to wide variability based on anatomic variations in the circle of Willis, ${ }^{7}$ these regional flows may provide a more reliable measure of hemodynamic status.

Many methods, including the nitrous oxide, ${ }^{23}{ }^{133}$ xenon (Xe)- single-photon emission CT (SPECT), ${ }^{9,17} \mathrm{Xe-CT},{ }^{24}$ positron-emission tomography $(\mathrm{PET})^{25,26}$ dynamic $\mathrm{MR},{ }^{25,27}$ and perfusion $\mathrm{CT},{ }^{24}$ have been used to measure the regional CBF. In most studies, ${ }^{9,17,23-27}$ CBF values were reported in terms of milliliters per 100 grams of brain tissue per minute, and in some studies CBFs were determined separately for white and gray matter. In a study of the effect of age on the total CBF using nongated 2D PC MRA, ${ }^{5}$ a factor of 10.8112.14 was used in the correlation between the total CBF (milliliters per minute) from the 2D PC MRA and the total CBF (milliliters per minute 100 grams) from the nitrous oxide, ${ }^{133} \mathrm{Xe}$-SPECT, Xe-CT, and PET. Our findings for the total $\mathrm{CBF}$, that is, total cerebral flow (IC ${ }^{\mathrm{r}}$ ), were comparable with those total CBF values from either gated or nongated 2D PC MRA. $^{3-5}$

A limitation of the present study was ophthalmic and middle meningeal artery flows were assumed to be negligible. Although this assumption may be reasonable for healthy volunteers, those volume flows could be significant in some patients with occlusive intracranial cerebrovascular disease and, therefore, must be taken into account in the calculation of the regional CBF. In addition, diseases like Moyamoya may be problematic to evaluate, because large vessels are typically replaced by multiple intracranial and extracranial collateral vessels that may not be amenable to flow measurement and would not be accounted for by the partitioning model. For the patient with an extracranial circulation-intracranial circulation bypass, ${ }^{28}$ the bypass flow must be measured and included in the regional flow calculation. Last, the number of subjects in each age group was small, especially in the oldest age group. This may limit our findings for the effect of age on both vessel flow and regional CBF.

In conclusion, regional CBF can be calculated using qMRA. The relative contribution of the subregions to the parent region, along with the regional flows, could provide useful information in the hemodynamic evaluation of patients with cerebrovascular disease.

\section{References}

1. Amin-Hanjani S, Du X, Zhao M, et al. Use of quantitative magnetic resonance angiography to stratify stroke risk in symptomatic vertebrobasilar disease. Stroke 2005;36:1140-45
2. Marks MP, Pelc NJ, Ross MR, et al. Determination of cerebral blood flow with a phase-contrast cine MR imaging technique: evaluation of normal subjects and patients with arteriovenous malformations. Radiology 1992;182:467-76

3. Enzmann DR, Ross MR, Marks MP, et al. Blood flow in major cerebral arteries measured by phase-contrast cine MR. AJNR Am J Neuroradiol 1994;15:123-29

4. Enzmann DR, Marks MP, Pelc NJ. Comparison of cerebral artery blood flow measurements with gated and ungated phase-contrast techniques. JMRI 1993;3:705-12

5. Buijs PC, Krabbe-Hartkamp MJ, Bakker CJ, et al. Effect of age on cerebral blood flow: measurement with ungated two-dimensional phase-contrast MR angiography in 250 adults. Radiology 1998;209:667-74

6. Boorder MJ, Hendrikse J, van der Grond J. Phase-contrast magnetic resonance imaging measurements of cerebral autoregulation with a breath-hold challenge: a feasibility study. Stroke 2004;35:1350-54

7. Bisschops RH, van der GraafY, Mali WP, et al. High total cerebral blood flow is associated with a decrease of white matter lesions. J Neurol 2004;251:1481-85

8. Hendrikse J, van Raamt AF, van der Graaf Y, et al. Distribution of cerebral blood flow in the circle of Willis. Radiology 2005;235:184-89

9. Ogasawara K, Ogawa A, Yoshimoto T. Cerebrovascular reactivity to acetazolamide and outcome in patients with symptomatic internal carotid or middle cerebral artery occlusion: a xenon-133 single-photon emission computed tomography study. Stroke 2002;33:1857-62

10. Kleiser B, Widder B. Course of carotid artery occlusions with impaired cerebrovascular reactivity. Stroke 1992;23:171-74

11. Kato T, Indo T, Yoshida E, et al. Contrast-enhanced 2D cine phase MR angiography for measurement of basilar artery blood flow in posterior circulation ischemia. AJNR Am J Neuroradiol 2002;23:1346-51

12. Guppy KH, Charbel FT, Corsten LA, et al. Hemodynamic evaluation of basilar and vertebral artery angioplasty. Neurosurg Clin N Am 2001;36:499-508

13. Perkio J, Soinne L, Ostergaard L, et al. Abnormal intravoxel cerebral blood flow heterogeneity in human ischemic stroke determined by dynamic susceptibility contrast magnetic resonance imaging. Stroke 2005;36:44-49

14. Neff KW, Horn P, Dinter D, et al. Extracranial-intracranial arterial bypass surgery improves total brain blood supply in selected symptomatic patients with unilateral internal carotid artery occlusion and insufficient collateralization. Neuroradiology 2004;46:730-37

15. Liu Y, Karonen JO, Vanninen RL, et al. Acute ischemic stroke: predictive value of 2D phase-contrast MR angiography-serial study with combined diffusion and perfusion MR imaging. Radiology 2004;231:517-27

16. Hagstadius S, Risberg J. Regional cerebral blood flow characteristics and variations with age in resting normal subjects. Brain $\operatorname{Cog} n$ 1989;10:28-43

17. Shirahata N, Henriksen L, Vorstrup S. Regional cerebral blood flow assessed by ${ }^{133} \mathrm{Xe}$ inhalation and emission tomography; normal values. J Comput Assist Tomogr 1985;9:861-66

18. Zhao M, Charbel FT, Alperin N, et al. Improved phase-contrast flow quantification by three-dimensional vessel localization. Mag Reson Imag 2000;186:697-706

19. Zhao M, Curcio AP, Clark ME, et al. In vitro validation of MR volumetric flow measurement. Proceedings of the 2004 International Workshop on Flow and Motion 2004:148-49

20. Charbel FT, Zhao M, Amin-Hanjani S, et al. Patient computer model to predict outcomes of the balloon occlusion test. J Neurosurg 2004;101:977-88

21. Ruland S, Zhao M, Pandey D, et al. Reproducibility of cerebral blood flow analysis using quantitative magnetic resonance angiography. Presented at the AANS/CNS Cerebrovascular Section 9th Joint Annual Meeting, February 1720, 2006; Orlando, Fla

22. Zhao M, Ruland S, Pandey D, et al. Repeatability of MR volumetric flow measurements in major cerebral arteries. Presented at 2006 ISMRM Flow and Motion Study Group Workshop: Imaging Assessment of Cardiovascular and Tissue Mechanics, July 13-16, 2006; New York, NY

23. Fazekas JF, Alman R, Bessman AN. Cerebral physiology of the aged. Am J Med Sci 1952;223:245-57

24. Wintermark M, Thiran JP, Maeder P, et al. Simultaneous measurement of regional cerebral blood flow by perfusion $\mathrm{CT}$ and stable xenon CT: a validation study. AJNR Am J Neuroradiol 2001;22:905-14

25. Carroll TJ, Teneggi V, Jobin M, et al. Absolute quantification of cerebral blood flow with magnetic resonance, reproducibility of the method, and comparison with $\mathrm{H} 2(15) \mathrm{O}$ positron emission tomography. J Cereb Blood Flow Metab 2002;22:1149-56

26. Pantano P, Baron JC, LeBrum-Grandie P, et al. Regional cerebral blood flow and oxygen consumption in human aging. Stroke 1985;15:635-41

27. Rempp KA, Brix G, Wenz F, et al. Quantification of regional cerebral blood flow and volume with dynamic susceptibility contrast-enhanced MR imaging. Radiology 1994;193:637-41

28. Chieregato A, Fainardi E, Servadei F, et al. Centrifugal distribution of regional cerebral blood flow and its time course in traumatic intracerebral hematomas. J Neurotrauma 2004;21:655-66 